\title{
A study on Proportion, Speciation and Antifungal Resistance Pattern of the Candida Isolates in a Tertiary Care Hospital of North Kerala, India
}

\author{
Katherine Joseph*, K.K. Ameena and Ann Taisy George \\ Department of Microbiology, MES Medical College, Perinthalmanna, \\ Malappuram-679338, India \\ *Corresponding author email id:
}

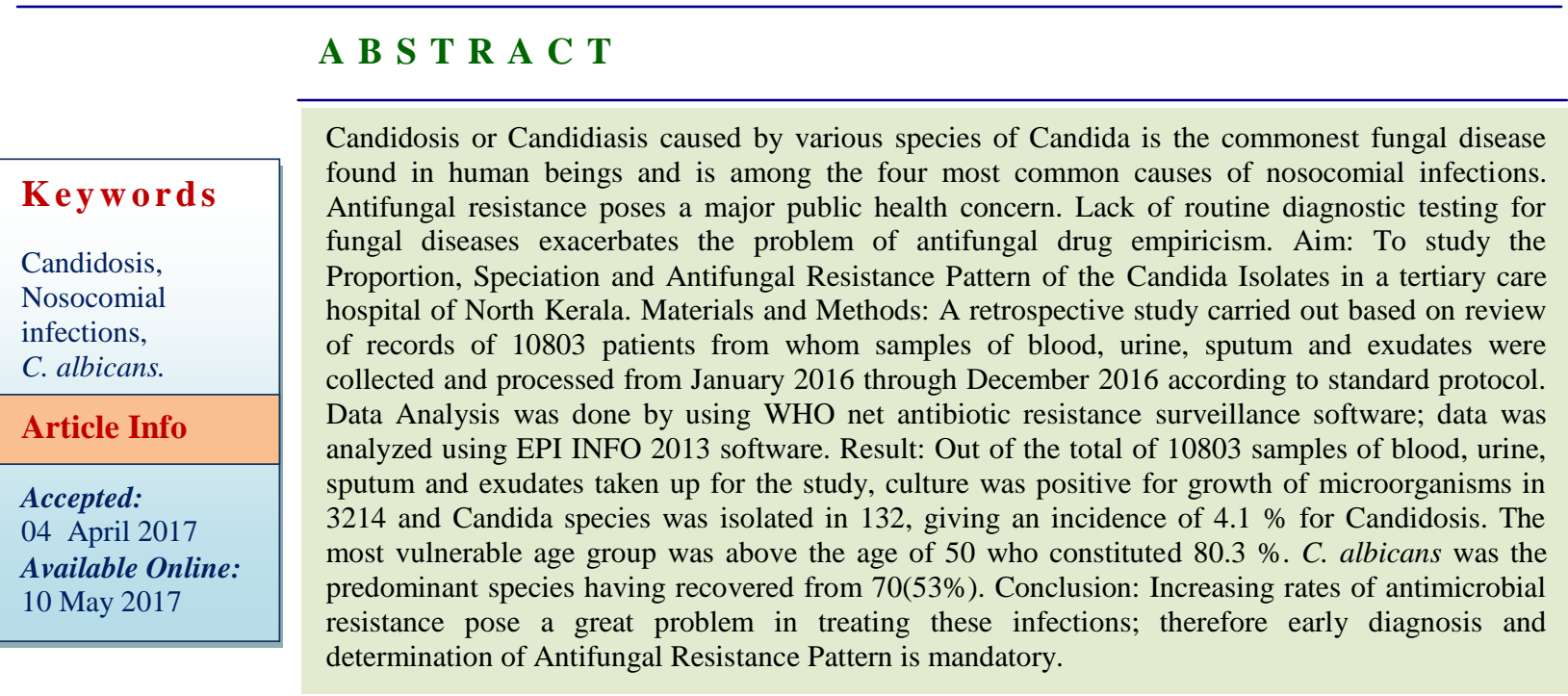

\section{Introduction}

Candidosis or candidiasis, caused by various species of Candida that are part of the normal flora of the muco-cutaneous membranes of humans, is the commonest fungal disease found in human beings. A variety of factors predispose to candidosis by altering the balance of normal microbial flora or by lowering the host resistance (Chander, 2013) resulting in tissue invasion and lifethreatening diseases in patients with decreased cell mediated immunity (Dharwad et al., 2011). The risk factors for candidosis include use of broad-spectrum antimicrobials, chemotherapy, invasive procedures, transplants and prolonged ICU stay. Most of the invasive infections due to Candida species are attributed to $C$. albicans, $C$. glabrata, $C$. parapsilosis, C. tropicalis and C. krusei (Giri et al., 2012; Ece et al., 2012); however accurate speciation is important for treatment, as not all species respond to the same treatment and due to anti-fungal resistance and emergence of non- albicans Candida species (Page et al., 2005). Candida species with fluconazole resistance have become more prominent in recent years (Chakrabarti et al., 1996). Early diagnosis and proper treatment is the key for management of candidosis. Hence this study was undertaken to determine the proportion of Candida 
species prevalent in our centre, to speciate them and to evaluate the resistance pattern to antifungal agents.

\section{Materials and Methods}

After getting approval from Institutional ethics committee, a retrospective study was carried out, based on review of records of 10803 patients from whom samples of blood, urine, sputum and exudates were collected and processed in the diagnostic section of Department of Microbiology, from January 2016 to December 2016, meeting inclusion and exclusion criteria. The basic sociodemographic information and data regarding age, gender, fungal isolates from samples of blood, urine, sputum and exudates and their antifungal resistance pattern were collected using predesigned Performa, according to standard protocol.

Urine, sputum and exudates were collected aseptically in sterile containers and transported without delay. The blood samples were collected aseptically and introduced into the BACTEC blood culture bottles immediately and the bottles shaken well. The samples were immediately placed in BACTEC automated blood culture system.

Urine, sputum and exudates were examined by wet mounts or $\mathrm{KOH}$ preparation and Gram stain. The samples were inoculated onto two tubes of Sabourauds Dextrose Agar (SDA) and one was incubated at room temperature and the other at $37^{\circ} \mathrm{C}$. All BACTEC positive samples were subjected to Gram stain and inoculated onto Blood agar plates and incubated at $37^{\circ} \mathrm{C}$ for 48 hours. Out of a total of 10803 various clinical specimens Candida isolates were obtained in 132 of them and their characterization and speciation was done based on cultural characteristics on SDA and CHROM agar, by the production of germ tubes, formation of chlamydoconidia on cornmeal agar, Gram stain and biochemical reactions for assimilation and fermentation of carbohydrates like glucose, sucrose and maltose (Bailey and Scott's, 2014). Antifungal susceptibility was done to determine the resistance pattern according to the CLSI guidelines by the disc diffusion method and the antifungal discs used are nystatin (100 units), amphotericin B $(20 \mu \mathrm{g})$, fluconazole $(25 \mu \mathrm{g})$, ketoconazole $(10 \mu \mathrm{g})$, voriconzole $(1 \mu \mathrm{g})$, and itraconazole $(10 \mu \mathrm{g})$.

Data Analysis was done by using WHO Net Antibiotic Resistance Surveillance Software; data was entered in Excel and analyzed using EPI INFO 2013 software.

\section{Results and Discussion}

Out of the total of 10803 samples of blood, urine, sputum and exudates taken up for the study, culture was positive for growth of microorganisms in 3214 and Candida species was isolated in 132, giving an isolation rate of $4.1 \%$ for Candidosis. Of these the incidence was $59.1 \%$ and $40.9 \%$ in male and female patients respectively (Figure 1). In relation to age factor, the most vulnerable age group was above 70 years where the incidence was $33.3 \%$ and those above the age of 50 constituted $80.3 \%$ (Figure 2).

Out of a total of 132 Candida isolates, $C$. albicans was recovered from $70(53 \%)$, followed by $C$. tropicalis in $23(17.4 \%) C$. parapsilosis in $13(9.8 \%)$ C. glabrata in 11 $(8.3 \%), C$. krusei in $7(5.3 \%)$ of patients (Figure 3).

C. albicans was the predominant organism in urine, sputum and exudates followed by $C$. tropicalis while in blood samples there was predominance of $C$. parapsilosis followed by $C$. albicans and $C$. glabrata (Table 1). The antifungal resistance pattern showed that the triazole antifungal agents like voriconazole, 
ketoconazole, fluconazole and itraconazole are more resistant to the Candida species isolated in this study than nystatin and amphotericin B. Voriconazole and ketoconazole exhibited $9.8 \%$ resistance, fluconazole $8.3 \%$ and Itraconazole $7.6 \%$; amphotericin B and nystatin showed 3.0\% and $2.8 \%$ resistance respectively (Table 2 ).

Candidosis is an opportunistic fungal infection caused by Candida species, a major component of human microbiota, acquired either endogenously or exogenously from sources in the healthcare system (Ahmad et al., 2012). A study by Hidron et al has reported that infections by Candida spp. are the fourth most common cause of device and procedure-associated nosocomial infections bloodstream infections in patients on central line, catheter-associated urinary tract infections, ventilator-associated pneumonia, and surgical site infections (Hidron et al., 2008). Antimicrobial resistance, a major public health concern, largely arises from excess use of antibiotic and antifungal drugs. Lack of routine diagnostic testing for fungal diseases exacerbates the problem of antimicrobial drug empiricism, both antibiotic and antifungal (Denning et al., 2017)

Table.1 Distribution of Candida species in various clinical specimens

\begin{tabular}{|l|l|l|l|l|l|}
\hline Candida species & \multicolumn{4}{|l|}{ Clinical specimen } & Total \\
\cline { 2 - 6 } & Blood & Urine & Sputum & Exudates & \\
\hline C. albicans & 2 & 30 & 26 & 12 & 70 \\
\hline C. tropicalis & 1 & 13 & 5 & 4 & 23 \\
\hline C. parapsilosis & 4 & 5 & 2 & 2 & 13 \\
\hline C. glabrata & 2 & 6 & 2 & 1 & 11 \\
\hline C. krusei & 0 & 5 & 0 & 2 & 7 \\
\hline Candida spp. & 1 & 3 & 3 & 1 & 8 \\
\hline Total & 10 & 62 & 38 & 22 & 132 \\
\hline
\end{tabular}

Table.2 Overall Antifungal resistance pattern of Candida isolates

$\mathrm{NS}=$ nystatin, $\mathrm{AP}=$ amphotericin $\mathrm{B}, \mathrm{KT}=$ ketoconazole, $\mathrm{FLC}-$ fluconazole, $\mathrm{VRC}=$ voriconazole, $\mathrm{IT}=$ itraconazole

\begin{tabular}{|l|l|l|l|l|l|c|}
\hline $\begin{array}{l}\text { Anti- } \\
\text { Fungal } \\
\text { Agents }\end{array}$ & $\begin{array}{l}\text { Blood } \\
(10)\end{array}$ & $\begin{array}{l}\text { Urine } \\
(62)\end{array}$ & $\begin{array}{l}\text { Sputum } \\
(38)\end{array}$ & $\begin{array}{l}\text { Exudate } \\
(22)\end{array}$ & $\begin{array}{l}\text { Total } \\
(132)\end{array}$ & Percentage \\
\hline NS & 0 & 1 & 0 & 2 & 3 & 2.8 \\
\hline AP & 0 & 1 & 1 & 2 & 4 & 3.0 \\
\hline KT & 1 & 6 & 4 & 3 & 13 & 9.8 \\
\hline FLC & 1 & 5 & 3 & 3 & 11 & 8.3 \\
\hline VRC & 1 & 6 & 4 & 3 & 13 & 9.8 \\
\hline IT & 1 & 5 & 3 & 2 & 10 & 7.6 \\
\hline
\end{tabular}


Figure.1 Age-wise prevalence

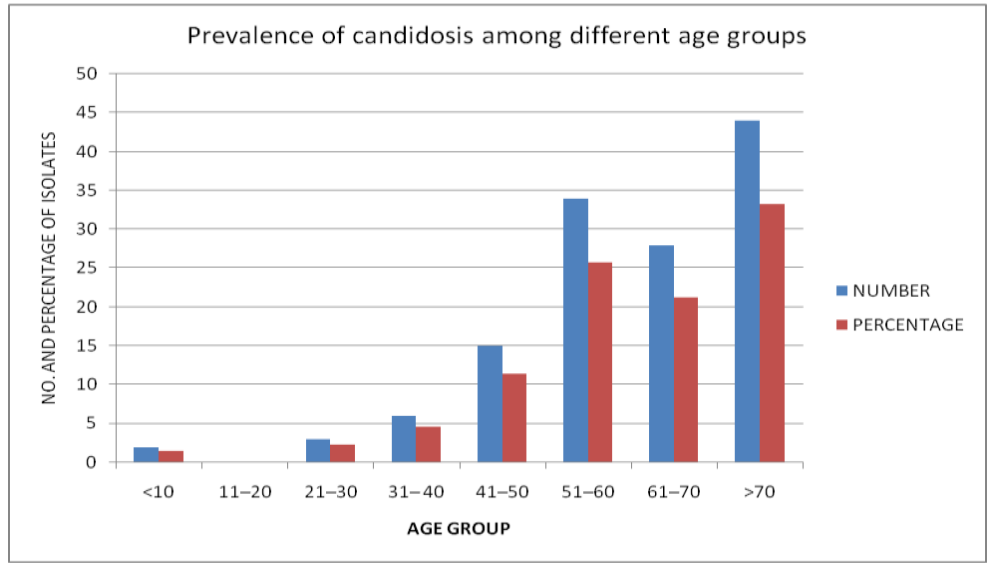

Figure.2 Gender-wise prevalence

Gender-wise prevalence of candidosis

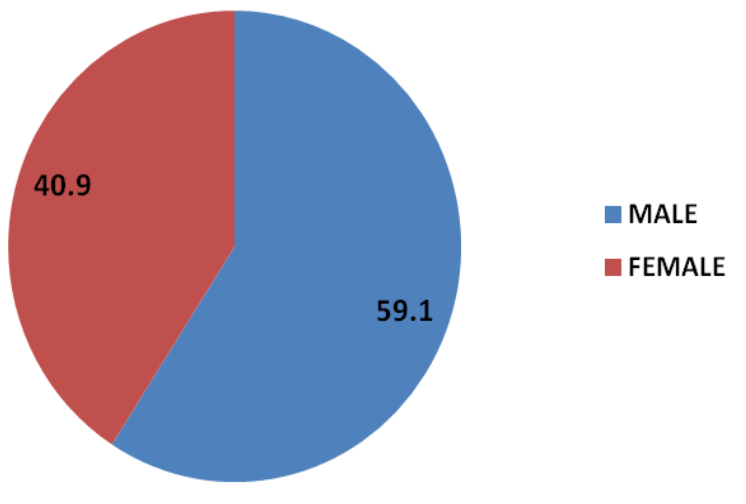

Figure.3 Species-wise distribution

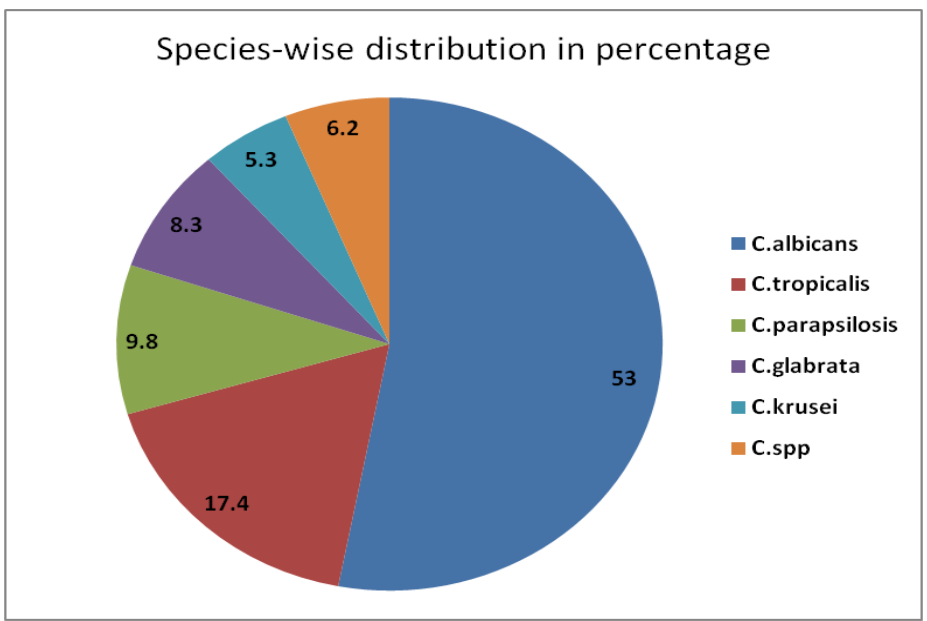


The gender-wise distribution of patients was $54.5 \%$ females and $45.5 \%$ males in a study by Guru et al., (2016) and in a similar study by Dharwad et al., (2011) also the incidence of candidiasis was higher in females $(64 \%)$ than in males $(36 \%)$. But in our study there is preponderance of male patients over females. Although candidosis can occur at all ages, studies by Dharwad et al., (2016) showed highest incidence of candidosis in the age group of 18 and 45(54\%) years followed by the age group of greater than 60 years $(22 \%)$. In a similar study by Dalal and Kelkar (1980) the highest incidence of candidosis was found to be in the age group of 21-40 years. In our study, the highest incidence was in patients above the age of 50 who constituted $80.3 \%$.

The risk factors reported by various authors include prolonged antibiotic therapy, catheterization, ICU stay, diabetes, malignancy, cancer chemotherapy, pulmonary diseases and central line and other devices in situ, sepsis, pregnancy, and immunosuppression including HIV (Singhal et al., 2015).

Bloodstream infection and invasive candidiasis are substantially more common than realized and probably result from multiple factors, including unrestrained antibiotic drug use, indwelling devices and increasing populations of immunocompromised patients. Multiple studies have shown the incidence of bloodstream infections with Candida spp. to be $1.2-26$ cases/100,000 population. (Denning et al., 2017) Distribution of Candida species in Candidosis vary from study to study. Although in most studies $C$. albicans remains the predominant species (Pfaller et al., 2007) a shift from C. abicans to non-albicans Candida too is being reported (Nguyen et al., 1996). In our study the predominant species was $C$. albicans having recovered from $70(53 \%)$, followed by $C$. tropicalis in 23 (17.4\%). High percentage of resistance against antifungal agent flucanozole by Candida species is being reported by various authors. In our study Voriconazole and ketoconazole were more resistant than the other agents tested.

In conclusion Candidosis caused by Candida species are on the rise and are among the four most common causes of nosocomial infections. Antifungal resistance poses a major public health concern. Lack of routine diagnostic testing for fungal diseases exacerbates the problem of antifungal drug empiricism. Health care professionals quite often face the challenge of drug resistance in patients on prolonged antibiotic therapy and in such a scenario should raise a high index of suspicion of the possibility of Candidosis and should get the collaboration of Clinical Microbiologist to arrive at a faster diagnosis aiming at improving the patient care.

\section{References}

Ahmad, S., Khan, Z. 2012. Invasive candidiasis: A review of non-culturebased laboratory diagnostic methods. Indian J. Med. Microbiol., 30(3): 264269.

Chakrabarti, A., Ghosh, A., Batra, R., Kaushal, A., Roy, P., Singh, H. 1996. Antifungal susceptibility pattern of nonalbicans Candida species and distribution of species isolated from candidaemia cases over a 5 year period. Indian J. Med. Res., 104: 171-6

Chander J. 2013. Textbook of Medical Mycology: $3^{\text {rd }}$ ed. Mehta Publishers, New Delhi: p. 266-90

Dalal, P.J., Kelkar, S.S. 1980. Clinical patterns of Candida infections in Bombay. Indian J. Dermatol. Venereol. Leprol., 46: 31-2.

Denning, D.W., Perlin, D.S., Muldoon, E.G., Colombo, A.L., Chakrabarti, A., 
Richardson, M.D., et al. 2017. Delivering on Antimicrobial Resistance Agenda Not Possible without Improving Fungal Diagnostic Capabilities. Emerging Infect. Dis., 23(2): 177-183.

Dharwad, S., Saldhana, D.R. 2011. Species identification of Candida isolates in various clinical specimens with their antifungal susceptibility patterns. $J$. Clin. Diagn. Res., 5(1): 1177-81

Ece, G., Samlioglu, P., Akkoclu, G., Atalay, S., Kose, S. 2012. The Evaluation of the Distribution of Yeast like Fungi 'Candida Species' at a Tertiary Care Center in Western Turkey. Int. J. Med. Sci., $\quad$ 9(7): $\quad$ 617-620. doi:10.7150/ijms.4707.

Giri, S., Kindo, A.J. 2012. A review of Candida species causing blood stream infection. Indian J. Med. Microbiol., 30(3): 270-8.

Guducuoglu, H., Gultepe, B., Otlu, B., Bektas, A., Yildirim, O., Tuncer, O., Berktas, 2016. M. Candida albicans outbreak associated with total parenteral nutrition in the neonatal unit. Indian J. Med. Microbiol., 34(2): 202-207.

Guru, P., Raveendran, G. 2016. Characterization and antifungal susceptibility profile of Candida species isolated from a tertiary care hospital. JACM, 18(1): 32-36:

Hidron, A.I., Edwards, J.R., Patel, J., Horan, T.C., Sievert, D.M., Pollock, D.A., et al.
2008. NHSN annual update: Antimicrobial-resistant pathogens associated with healthcare-associated infections: Annual summary of data reported to the National Healthcare Safety Network at the Centers for Disease Control and Prevention, 20062007. Infect. Control Hosp. Epidemiol., 29: 996-1011.

Nguyen, M.H., Peacock, J.E., Jr, Morris, A.J., Tanner, D.C., Nguyen, M.L., Snydman, D.R., et al. 1996. The changing face of candidemia: emergence of non-Candida albicans species and antifungal resistance. Am. J. Med., 100(6): 617-23.

Page, B.T., Kurtzman, C.P. 2005. Rapid identification of Candida species and other clinically important yeast species by flow cytometry. J. Clin. Microbiol., 43: 4507-14

Patricia, M. Tille. 2014. The yeasts. In: Bailey and Scott's Diagnostic Microbiology: $13^{\text {th }}$ ed. Elsevier Mosby St. Louis, Missouri, 63043: 771-782

Pfaller, M.A., Diekema, D.J. 2007. Epidemiology of invasive candidiasis: A persistent public health problem. Clin. Microbiol. Rev., 20: 133-63.

Singhal, A., Sharma, R., Meena, V.L., Chutani, A. 2015. Urinary Candida isolates from a tertiary care hospital: speciation and resistance patterns. $J$. Acad. Clin. Microbiol., 17: 100-5.

\section{How to cite this article:}

Katherine Joseph, K.K. Ameena and Ann Taisy George. 2017. A study on Proportion, Speciation and Antifungal Resistance Pattern of the Candida Isolates in a Tertiary Care Hospital of North Kerala. Int.J.Curr.Microbiol.App.Sci. 6(5): 434-439. doi: http://dx.doi.org/10.20546/ijcmas.2017.605.050 